\title{
Figural vs. configural effects in the filled duration illusion
}

\author{
LYNN C. ROBERTSON and LOUIS M. GOMEZ \\ University of California, Berkeley, California 94720
}

\begin{abstract}
Patterns varying in form goodness and size were presented for 15,30 , and $45 \mathrm{msec}$. The experiment included three independent groups of subjects. Half of the first group were presented size variations with good figures, while the other half were shown size variations with poor figures. Half of the second group were presented goodness variations with small figures, while the other half were shown goodness variations with large figures. A third group experienced variations in both goodness and size. The major findings were: (1) poor figures and large figures were judged "longer" than good or small figures when varied within a session; (2) this effect vanished when the different levels of size or goodness were presented to separate groups of subjects; (3) the magnitude of the difference in temporal estimation was the same for size and goodness when each was varied in isolation; (4) when size and form goodness were varied orthogonally in the same session, both dimensions produced reliable changes in temporal estimation. These data are discussed in light of current theoretical explanations of the filled duration illusion.
\end{abstract}

When subjects are asked to judge the duration of an experimentally presented time interval, they will respond differently, depending on the number and/or type of events that occur during the interval (e.g., Block, 1974; Buffardi, 1971; Cantor \& Thomas, 1977; Ornstein, 1969). For instance, if one interval contains a complex stimulus and a second interval of the same length contains a simple stimulus, the interval filled with the complex stimulus will be judged longer than the interval filled with the simple stimulus. This effect is known as the filled-duration illusion.

Theoretical attempts to explain this phenomenon have localized the illusion at various stages during the processing of information. Cantor and Thomas and Thomas and Weaver (1975) have suggested that the illusion occurs because duration estimation is based on the processing time and attention needed to encode the nontemporal information which fills the experimental interval. This model relies on the assumption that it takes more time to encode larger or more complex events than it does to encode smaller or simpler events. Ornstein (1969) has claimed that the "storage space" needed to represent the nontemporal stimulus is the functional parameter in estimating the duration of any filled interval. His model assumes that larger or more complex events require more "mnemonic space" than smaller or simpler events,

This work was partially supported by USPHS training Grant gm1207 to the present authors through the Institute of Human Learning. Reprint requests should be sent to Lynn C. Robertson at the Institute of Human Learning, University of California, Berkeley, California 94720. We wish to thank Stephen Palmer for his support and critical comments concerning this research. Louis M. Gomez is now at Bell Laboratories, Murray Hill, New Jersey. and that temporal judgments vary monotonically with amount of space required. Thus, more storage space produces longer temporal judgments.

Gomez and Robertson (1979) and Robertson and Gomez (Note 1) have questioned these interpretations. They have pointed out that both theories share the assumption that the illusion results from absolute, rather than relative, characteristics of each stimulus event. The encoding model assumes that the relevant characteristic is the amount of processing time, and the storage model assumes that it is the number of informational units. Gomez and Robertson tested the hypothesis that larger stimulus items have an inherent, absolute property which makes them appear to have been presented longer than smaller stimulus items. In their experiments, each subject was presented one of three intervals per trial. Each interval was filled with either large or small stimuli. When the set of three intervals was quite discriminable, the illusion (durations filled with larger stimuli judged longer than durations filled with smaller stimuli) was significantly reduced as compared to a condition where the set was difficult to discriminate. This suggests that the illusion may occur only under conditions of uncertainty. Furthermore, when one group of subjects saw only one sized stimuli at the three durations and another saw the other sized stimuli, the illusion was eliminated regardless of the temporal range. Thus, the context in which the stimulus is embedded seems to be the important variable in producing an illusion.

In order to accommodate these results, proponents of the models outlined above would have to make substantial changes in their theories. It could be argued, however, that the size manipulation in the Gomez and Robertson study is unsuitable for the 
investigation of models based on complex cognitive constructs. The storage model explains the filled duration illusion in terms of the information content of a stimulus item. It is questionable whether increasing the size of a figure increases the amount of information in the stimulus. The encoding model also relies on information content as it relates to processing time, but it allows for other variations to affect processing time as well. Thomas and Cantor (1975) have proposed a sequential sampling process which produces increased encoding time with increased area. This process does not necessarily increase storage requirements. The encoding model, then, allows for temporal increases as a function of information or area. The null effects which occur when varying size between subjects might, therefore, be placed into the encoding model framework by appealing to sampling adjustments. Subjects seeing the same sized stimuli that do not vary in information content may adjust the size of their "sampling window" accordingly (i.e., adjust how much area is monitored in one sample). However, if a dimension that varies information content produces the same results as the size dimension, the premises underlying the "sampling window" adjustment argument are not tenable. A stimulus with more information should require more processing whether seen in the context of stimuli with less information or in isolation.

In the present study, two levels of size and two levels of goodness (an information manipulation) were presented in the same general paradigm as used in the previous Gomez and Robertson study. In one condition, size was varied orthogonally with goodness within a session to look at how subjects utilize multidimensional stimuli in their temporal judgments. Few investigators have studied the role played by multiple sources of variation in the temporal illusion. When they have done so, the concurrent presentation has not been a focus of attention (Avant \& Lyman, 1975; Warm \& McCray, 1969).

An additional goal of the present research is to determine whether figural goodness produces an illusion when other more separable attributes of form such as number of angles and the ratio of area to perimeter are held constant (e.g., Cantor \& Thomas, 1977). Although these individual attributes have been shown to produce the illusion, configural effects have not been fully examined. Subjects could be reacting to more wholistic aspects of the figure rather than to its local elements.

In summary, the present experiment is directed to (1) an extension of the previous size results by using an experimental factor which clearly manipulates information, (2) an examination of the effects of bidimensional variation on temporal judgments, and (3) the investigation of wholistic stimulus properties on time judgments.

\section{METHOD}

Subjects

The subjects were 24 undergraduates at the University of California, Berkeley. They participated in partial fulfillment of an introductory psychology course requirement.

\section{Materials}

Four patterns were constructed. Two of the patterns had a high value on a pattern goodness scale, and the two other patterns had a relatively low value on the pattern goodness scale. Each of the four patterns occured in two sizes, resulting in a total of eight figures, four of which were large and four of which were small. Examples of goodness and size are shown in Figure 1. Pattern goodness was defined according to Garner's (1974) rotation and reflection (R\&R) subsets formulation, which refers to the number of different transformations a figure generates by 90 -deg rotations in the picture plain plus reflections about the vertical and horizontal axes. Patterns with small R\&R subsets are high in figural goodness, while patterns with large $R \& R$ subsets are low in figural goodness. In the current study, the patterns with high figural goodness had an R\&R subset value of one and were low in informational content, and patterns with low figural goodness had an R\&R subset value of eight and were high in informational content. The smaller figures were 4/9 the size of the larger. The large figures subtended approximately 4 deg of visual angle, while the small figures subtended approximately $2.5 \mathrm{deg}$.

\section{Design and Procedure}

Goodness and/or size was varied as a within- or between-subjects factor. That is, one-third of the subjects saw only large or only small figures that varied in figural goodness (Group G); one-third of the subjects saw only good or only poor figures that varied in size (Group S); and the remaining third saw patterns that varied orthogonally in pattern goodness and size (Group GS). All patterns in all conditions were presented at three exposure durations: 15,30 , and $45 \mathrm{msec}$, which were called "short," "medium," and "long" by the subjects.

Stimuli were presented, one figure per trial, on an Iconix threefield tachistoscope. The illumination was constant. Each trial first contained a 550-msec "warning" flash telling a subject that the
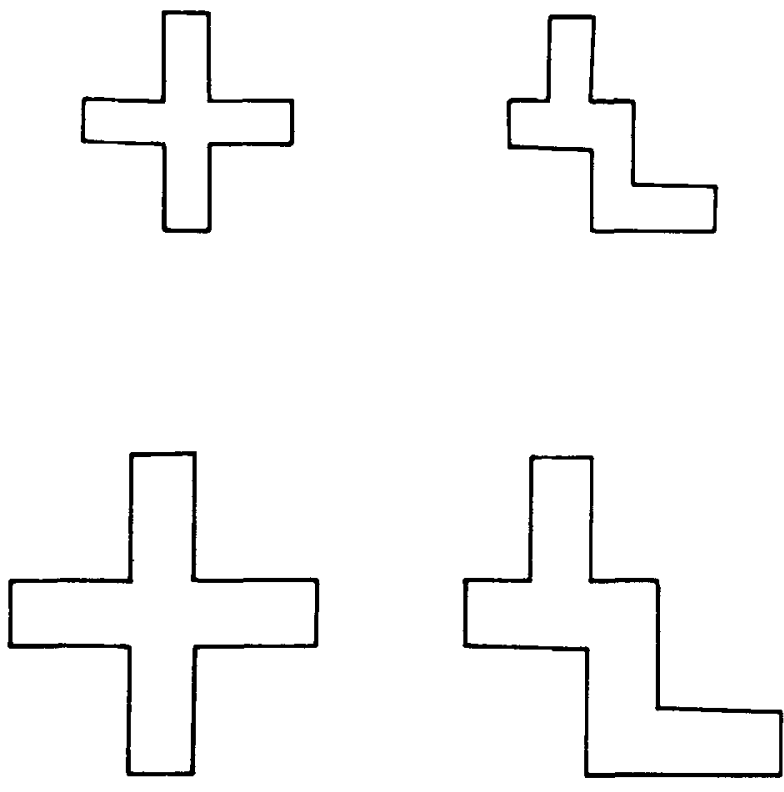

Figure 1. Example of stimulus figures. 

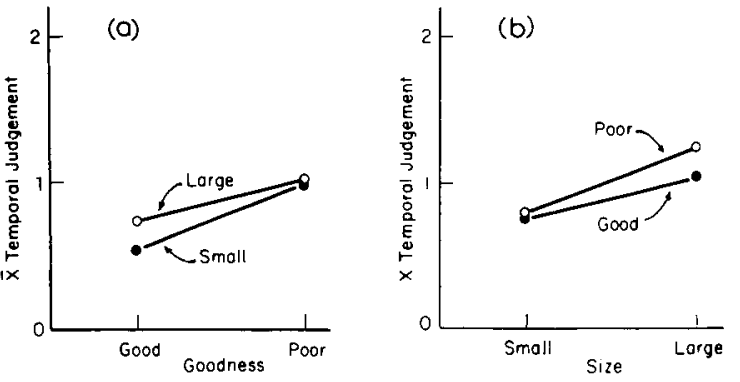

Figure 2. Mean temporal judgment with size as a betweensubject variable and goodness a within-subject variable (a), and with goodness as a between-subject variable and size a withinsubject variable (b).

trial had begun. Five hundred milliseconds after termination of the warning flash, a pattern flash was given for a duration corresponding to the particular condition and trial. A dark period of 500 msec followed the pattern and was ended by the advent of a display with the words SHORT, MEDIUM, and LONG written on it.

Experimental stimuli were presented in four blocks of 24 . For Group GS, the entire set of eight stimuli was randomly shown, once at each of the three durations in each block of trials. For Group $S$ and $G$, each stimulus in the relevant set of four was shown twice at each of the three durations within a block of 24. The presentation of blocks was newly randomized for each subject.

Before the experimental trials began, the subjects were given 30 practice trials with $15-, 30-$, and $45-$ msec durations filled with a retangular figure. The dimensions of this figure were approximately halfway between the two sizes used in the within-subjects experimental trials. The three durations were presented randomly, and feedback was given. Feedback was terminated at the end of practice. Following practice, 12 filler trials were presented. Filler trials were not included in the analysis.

Subjects were instructed that they would be making judgments about the pattern duration. They were told that each pattern would occur for 15,30 , or $45 \mathrm{msec}$ in randomly presented trials and that they should clearly state whether the duration was "short," "medium," or "long" when the final display appeared. No mention was made of pattern variation. When the subjects had finished responding, they were told to press a panel switch on a table in front of them. This switch turned off the final display light and reset the equipment for a new trial.

\section{RESULTS}

To quantify the dependent variable, the values of 0,1 , and 2 were given to the responses short, medium, and long (short $=0$, medium $=1$, long $=2$ ). A mean response value was then obtained for each subject for each duration for each level of the nontemporal variable. These were then analyzed using analysis of variance techniques.

When stimulus size was a between-groups variable and goodness a within-subjects variable (Group G), no duration illusion resulted as a function of size $[F(1,6)=2.04$, n.s. $]$, but a significant duration illusion was produced as a function of goodness $[F(1,6)$ $=26.60, \mathrm{p}<.001]$ (Figure $2 \mathrm{a}$ ). When subjects were exposed to the stimulus-size dimension as a withingroups variable while goodness was varied between groups (Group S), size produced a reliable duration illusion $[F(1,6)=11.25, p<.001]$ and goodness failed to show an illusion $[F(1,6)=1.34$, n.s.] (Figure $2 b$ ). Figure 2 shows clear effects of the nontemporal context on the filled-duration illusion. There are marked increases in temporal judgment as a function of the within-subject variable. (Notice that the $\mathrm{x}$-axes correspond to the within-subjects factor). The differences that appear in Figure 2a (Group S) for good figures and in Figure $2 b$ (Group $G$ ) for large figures were not significant $[F(1,6)=2.87$, n.s., for Group $S$ (Winer, 1971, method of planned comparisons); $F(1,6)$ $=1.98$, n.s., for Group G].

An analysis was performed to test for a possible interaction between the functions plotted in Figures $2 \mathrm{a}$ and $2 \mathrm{~b}$. The repeated measures factor (size or goodness) by between-groups factor (size or goodness) by levels $(1=$ good or small, 2 = poor or large) analysis resulted in a nonsignificant three-way interaction $[F(1,12)=1.73$, n.s.]. A nonsignificant Repeated Factor by Between Groups Factor interaction suggested that there was no difference between the magnitude of the illusion as a function of the type of variable that was used to produce it. To test this more directly, difference scores were computed for each subject for Group $G$ and Group S. A t test revealed that the size of the illusion in Group $S$ did not significantly differ from the size of the illusion in Group $\mathrm{G}[\mathrm{t}(14)=.04$, n.s.].

When the pattern size and figural goodness factors were orthogonally combined and presented to

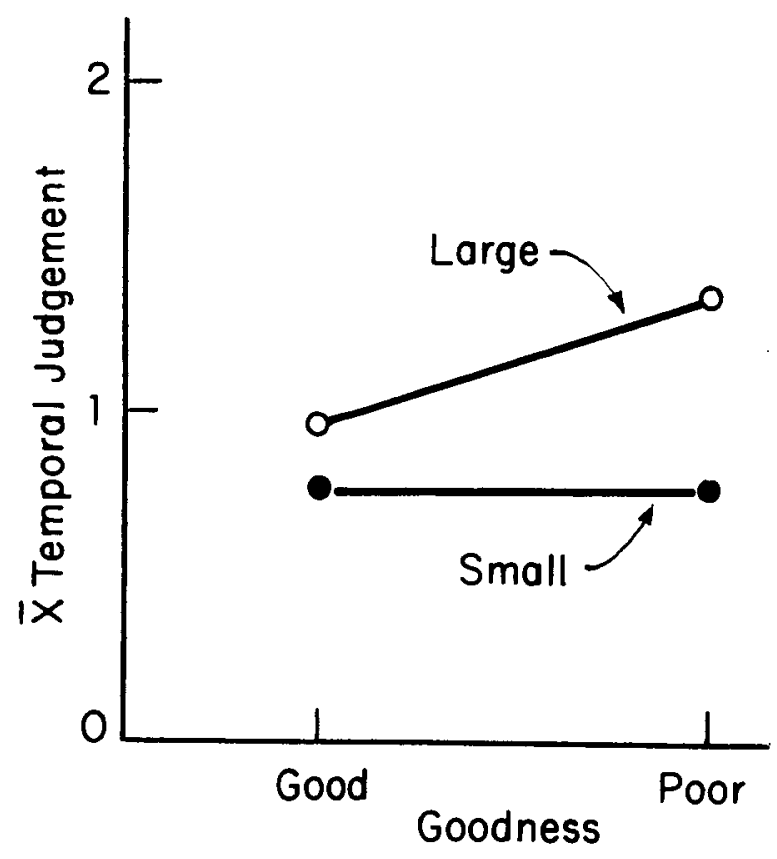

Figure 3. Mean temporal judgment with both goodness and size as within-subject variables. 


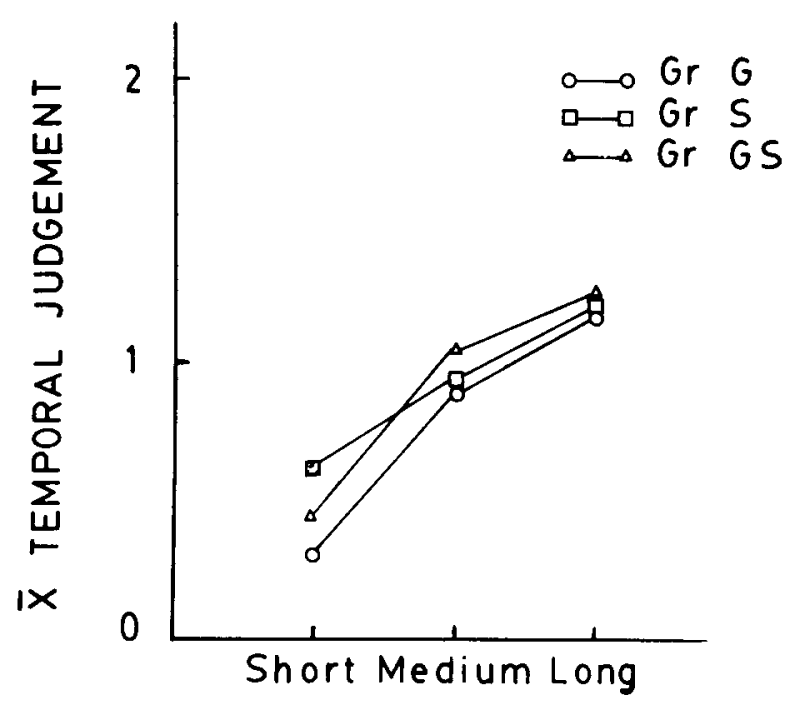

PRESENTED DURATION

Figure 4. Mean temporal judgment for each of the three groups as a function of presented duration (collapsed over size and goodness).

the same subjects, both factors had reliable effects on duration judgments. Figure 3 depicts the effect of size $[F(1,7)=37.00, p<.001]$ and the effect of goodness $[F(1,7)=7.10, p<.05]$. The change produced in duration judgments by a completely withinsubjects design was not the same for both factors, resulting in a significant Size by Goodness interaction $[F(1,7)=13.64, p<.01]$. For good figures, the size effect was not significant $[F(1,7)=2.73$, n.s.], nor was there a significant effect of goodness at small sizes $(F<1)$. Difference scores were computed for each subject in Group GS. The illusion for size was significantly greater than the illusion for goodness $[t(7)=2.40, p<.05]$.

A trend analysis was performed by collapsing over the size and goodness factor. The groups (G, S, GS) by duration analysis resulted in a significant linear trend $[F(1,21)=39.17, p<.001]$, which did not interact with groups $(F<1)$. Figure 4 expresses the linear increase in temporal judgments as the presentation duration increased.

\section{DISCUSSION}

The results support the Gomez and Robertson (1979) findings. The dimensions of goodness and size both produce an illusion when varied as within-subject variables. These effects are eliminated in both cases when varied between subjects. The results in the present study do show a slight tendency for large and poor figures to be judged longer than small or good figures. This tendency was not statistically significant and was not evident in the previous studies using only size, where, if anything, the trend was reversed.

The results demonstrate that a dimension assumed to contain informational variation (goodness) and one assumed to contain little, if any, informational variation (size) produce the same magnitude of the illusion when a second dimension is not also varied within the same session. This finding poses problems for both Ornstein's storage model and the Thomas and Cantor encoding model. Differences between size and differences between goodness appear to be processed differently. Larson and Bundesen (1978) have shown that the time to match two larger figures is virtually the same as matching two smaller figures. However, evidence from Garner's laboratory (Garner, 1974) supports the contention that figures with an $R \& R$ value of 8 take significantly longer to process for figural identity than figures with an $R \& R$ value of 1 . Thus, the encoding model should predict that goodness would produce a larger illusion than size. The present design produces the opposite effect. The storage model can, on the other hand, allow for an effect of size on temporal judgments only if it concludes that large-sized stimuli take more "mnemonic space" than small-sized stimuli. Gomez and Robertson (1979) suggested that a strategy may be imposed under conditions of temporal uncertainty, and that this strategy is linked to a nontemporal stimulus dimension. It is an important part of the present findings that the null magnitude result in the between-groups design concurs with this approach. It is the subject's ability to discriminate between durations that changes the probability of utilizing a dimension other than time. Discrimination of duration or duration uncertainty should be constant in the two groups, and the nontemporal dimension, whether goodness or size, would have a similar effect. This, in fact, is what occured.

In most studies of duration perception, temporal uncertainty has been induced by either a retrospective design in which subjects are not told in advance that they will be asked for temporal judgments or by the use of temporal variations that are difficult to discriminate. The strategy hypothesis suggests that under such conditions a subject will integrate the more discriminable, nontemporal variation with the temporal variation. For example, large stimuli will be judged longer in temporal extent because "large" and "long" are "more" of the respective dimensions, while "small" and "short" are "less" of the dimensions.

There is some evidence that there is a directional ease in processing a stimulus item relative to other directionally opposite stimuli. Clark and Chase (1972) found that subjects were faster at saying a star was above a plus than that a plus was below a star. Even though the stimulus item was the same, the state of being "above" seemed to be easier to process 
than the state of being "below." Findings like this have led to the suggestion that subjects "mark" a dimension. In the case of size and time, the hypothesis is that subjects mark the size of a stimulus item in terms of "largeness" and "longness." To the subject, size begins at zero and increasingly gets larger. It does not begin at infinity and increasingly get smaller. Likewise, duration begins at zero and increasingly gets longer. It does not begin at infinity and increasingly get shorter. Information could be represented in the same fashion. Thus, the contention is that larger sizes are thought of as "more large" rather than "less small," that poor figures are thought of as "more information" rather than "less noninformation," and that longer intervals are considered "more long" rather than "less short."

The strategy hypothesis proposes that the directional marking allows responses from a more discriminable dimension to be substituted for a less discriminable dimension in a manner where the marked directions of both dimensions correspond. It is clear that the strategy hypothesis is based on the relative information in the experimental environment, whereas the storage and encoding models are not. The difference in temporal judgments between the within-subjects and between-subjects groups is consistent with a strategy-based approach.

A result that is not completely consistent with this hypothesis is the Goodness by Size interaction when both dimensions are varied within the same session (Figure 3). The full implications of this interaction are not clear, but it does suggest that subjects are sensitive to variations on multiple dimensions. While this interaction shows that duration judgments affected by one dimension (the between-subjects results) can be changed by the inclusion of another dimension (the within-subjects results), the manner in which multiple or single sources of variation are applied to duration judgments has not been clarified. The fact that subjects demonstrate an illusion effect of both variables does not preclude a strategic basis for the filled-duration illusion. It does, however, suggest that the strategy is more complex than a simple one-for-one substitution of a nontemporal dimension for the temporal dimensions when more than one dimension is varied in the stimulus set.

Previous studies have shown the systematic nature of the nontemporal influence on temporal judgmentshigher values of a nontemporal dimension produce longer duration judgments then lower values. When two dimensions are varied, other, more complicated, results occur. Warm and McCray (1969) varied two levels of word length and two levels of word frequency in a repeated measures design. Low-frequency words with more letters produced shorter duration judgments than any of the other three conditions, the latter being essentially equal. The Word Length by Word Frequency interaction in that study and the Goodness by Size interaction in the present study must be examined more fully before a strategy-based model can be delineated.

A finding in the Warm and McCray study which also poses problems for the strategy hypothesis is that for low-frequency words, fewer letters rather than more letters produced longer judgments. This finding, however, is contrary to numerous other studies that have manipulated a spatial dimension in various forms (Buffardi, 1971; Cantor \& Thomas, 1977; Mo, 1971; Mo \& Michalski, 1972; Thomas \& Cantor, 1975). The results of the Warm and McCray study need clarification. A reasonable first step would be to manipulate word length without the inclusion of word frequency.

Finally, the results of the present investigation show that the filled-duration illusion can be produced when complexity is measured by configural properties. Previous research has shown that the complexity of an object can produce the illusion phenomenon (Cantor \& Thomas, 1977; Ornstein, 1969). Complexity, however, has typically been measured by counting the discrete segments of the stimulus event. The more parts an event contains, the more complex it is. The present work demonstrates that subjects respond to and utilize the wholistic properties of a stimulus in performing a temporal task.

In summary, the present research has shown that when one dimension is varied, the magnitude of the filled-duration illusion is unchanged by the nature of that dimension. In addition, this experiment has replicated previous work in that the illusion effect does not take place as a function of a given dimension when the values on that dimension are held constant within a session. The suggestion is that these results may be due to a strategy utilizing the nontemporal dimension. Although the within-subject data in the present experiment do not support a simple strategy model as suggested by Gomez and Robertson (1979), neither do they negate a model based on more complex strategic processes. The attraction of a stragety-based model is that, unlike encoding and storage models, it places a great deal of weight on the observer's perception of change. The current results demonstrate the importance of considering this parameter. It is interesting to note that Block and Reed (1978) are converging on a similar theoretical account while trying to develop an explanation for the effect of memory on retrospective time judgments.

\section{REFERENCE NOTE}

1. Robertson, L. C., \& Gomez, L. M. Perceived duration as a function of temporal and non-temporal stimulus set. Paper presented at the Western Psychological Association, San Francisco, 1978. 


\section{RETERENCES}

Avant, L. L., \& Lyman, P. J. Stimulus familiarity modifies perceived duration in prerecognition visual processing. Journal of Expetimental Psychology: Human Perception \& Performance, $1975,1,205-213$.

BLock, R. A. Memory and the experience of duration in retrospect. Perception \& Psychophysics, 1974, 2, 153-160.

BLock, R. A., \& REeD, M. A. Remembered duration: Evidence for a contextual-change hypothesis. Journal of Experimental Psychology: Human Learning and Memory, 1978, 4, 656-665.

Buffardi, L. Factors affecting the filled duration illusion in the auditory, tactual, and visual modalities. Perception \& Psychophysics, 1971, 10, 292-294.

Cantor, N. E., \& Thomas, E. A. C. The control of attention in the processing of temporal and spatial information in complex visual patterns. Journal of Experimental Psychology: Human Perception \& Performance, 1977, 3, 243-250.

Clark, H. H., \& Chase, W. On the process of comparing sentences against pictures. Cognitive Psychology, 1972, 3, 472-517.

Garner, W. R. The processing of information and structure. Potomac, Md: Erlbaum, 1974.

Gomez, L. M., \& Robertson, L. C. The filled duration illusion: The function of temporal and non-temporal set. Perception \& Psychophysics, 1979, , 25, 432-438.
Larson, A., \& Bundesen, C. Size scaling in visual pattern recognition. Journal of Experimental Psychology: Human Perception and Performance, 1978, 4, 1-20.

Mo, S. S. Judgment of temporal duration as a function of numerosity. Psychonomic Science, 1971, 24, 71-72.

Mo, S. S., \& Michalski, V. A. Judgment of temporal duration of area as a function of stimulus configuration. Psychonomic Science, 1972, 27, 97-98.

Ornste in, R. E. On the experience of time. Middlesex, England: Penguin Books, 1969.

Thomas, E. A. C., \& Cantor, N. E. On the duality of simultaneous time and size perception. Perception \& Psychophysics, $1975,18,41-48$.

Thomas, E. A. C., \& Weaver, W. B. Cognitive processing and time perception. Perception \& Psychophysics, 1975, 17, 363-367.

WARM, J. S., \& McCraY, R. E. Influence of word frequency and length on the apparent duration of tachistoscopic presentations. Journal of Experimental Psychology, 1969, 79, 56-58.

WINER, B. J. Statistical principles in experimental design. New York: McGraw-Hill, 1971.

(Received for publication April 16, 1979; revision accepted October 30, 1979.) 